WIENER SLAVISTISCHES JAHRBUCH, Band 53/2007, 37-53

(C) 2007 by Österreichische Akademie der Wissenschaften, Wien

GERHAR D NEWEKL OW S K Y

\title{
Primož Trubar als Schöpfer einer Schriftsprache
}

\begin{abstract}
Prispevek raziskuje, kako je Trubar svoj leta 1550 utemeljeni knjižni jezik do leta 1566 razvijal in utrdil in kako so mlajši avtorji (Duhovne peisni, Krelj, Dalmatin, Bohorič) njegove rešitve prevzeli ali izboljšali. Konkretno je obravnavano: pisanje $l l: l$, načini pisanja $s$-ja in $\breve{s}$-ja, refleksi jata, preglas za zgodovinsko mehkimi soglasniki, samostalniške končnice v dajalniku množine, zvočnika lj nj, predlogi na za nad raz, uvedba naglasnih znakov, kratke oblike pridevnikov, določni členek, sklanjatve tipa bog in srce, pogostost rabe sklonov in sklanjatev, nadaljnje posebnosti sklanjatve in spregatve. Avtor pokaže, da je Trubar sam v teku let svoje rešitve izboljšal in normiral in da so mlajši avtorji in revizijska komisija iz leta 1581 modificirali samo nekatere njegove odločitve.
\end{abstract}

Der Titel meines Beitrags ist eine Paraphrase des Buchtitels Primus Truber Schöpfer der slovenischen Schriftsprache? von Elisabeth Seitz (Seitz 1998). Die Autorin hat vor allem an der Syntax gezeigt, wie Trubar diese schriftsprachlich stilisiert und auf diese Weise die slowenische Schriftsprache tatsächlich geschaffen hat.

Wir wollen in diesem Aufsatz versuchen, der Sprache Trubars zwischen 1550 und 1566 nachzugehen und dabei besonders die Frage der Normierung seiner Sprache verfolgen sowie sie mit der Sprache jüngerer Schriftsteller vergleichen. Eine Schriftsprache kann nur dann als solche funktionieren, wenn sie einigermaßen normiert ist und wenn andere Schriftsteller sich an die vorgegebenen Normen halten.

\section{Die VorausSETZUngen}

Über Trubars Leben sind wir ausgezeichnet informiert, ebenso verfügen wir über zahlreiche Arbeiten zu den kulturgeschichtlichen Hintergründen der Zeit des Protestantismus, aber auch über Trubars Sprache. So wissen wir sehr gut, wie es dazu gekommen ist, dass sich Trubar im Jahre 1550 seinen lang gehegten Wunsch erfüllen konnte, das erste slowenische Buch zu schreiben und zu veröffentlichen: Es war dies der Catechifmus In der Windischenn Sprach mit dem slowenischen Untertitel „Anu kratku Poduuzhene Ikaterim vfaki zhlouik more vnebu pryti“. Gedruckt wurde das Buch in Tübingen bei Ulrich Morhart, der damals allerdings seinen Namen nicht nennen durfte, und so wählte Trubar das Pseudonym Janez Skuryanez für seinen 
Drucker, und für sich selbst Philopatridus illyricus. Das erste Buch beinhaltete den Katechismus, Kirchenlieder, die Litanei, zwei Kollektengebete und eine längere Predigt (in deren Titel Trubars Name genannt wird); das Buch besteht also aus heterogenen Teilen, die sich auch sprachlich deutlich voneinander unterscheiden (Neweklowsky 1985). Kurz nach dem Katechismus erschien das Abecedarium und der klein Catechifmus In der Windischen Sprach (1550), eine Notwendigkeit, wenn Trubar damit rechnete, dass auch andere Personen Slowenisch lesen und schreiben lernen sollten. Wir wissen, dass Trubar lange Jahre slowenischer Prediger war und sich dabei Notizen in slowenischer Sprache machen konnte, wenngleich dies nicht sicher ist. Nach seinen Aussagen sei die slowenische Sprache vor K 1550 weder geschrieben noch gedruckt worden (vgl. Toporišič 1983/84: 227). Freilich wissen wir nicht, ob seine diesbezüglichen Aussagen wirklich wörtlich zu nehmen sind.

1555 wurde der Augsburger Religionsfriede geschlossen, in dessen Folge es zu einem Mangel an protestantischen Geistlichen kam, so dass sich Trubar seinen Arbeitsplatz aussuchen und Kempten im Allgäu wählen konnte. In jene Zeit fällt auch Trubars neuerliche Begegnung mit Vergerius, der 1553 als Ratgeber Herzog Christophs nach Württemberg gegangen war. Er war es, der Trubar dazu überredete, sich an die Übersetzung der Heiligen Schrift zu machen (Rupel 1965: 97-101). In demselben Jahr gab Trubar sein zweites Abecedarium und seine zweite Version des Katechismus (Catechismus v slovenskim jeziku $=\mathrm{K} 1555$ ) heraus. Im Abecedarium verließ Trubar die Schwabacher Fraktur und ging zur Lateinschrift im slowenischen Text über. Er begann seine Übersetzertätigkeit mit dem Matthäusevangelium, ebenfalls 1555 (= M 1555). Dies war das erste Buch, das allein in slowenischer Sprache geschrieben wurde. In seinem Vorwort finden wir die erste bekannte Stellungnahme über die dialektale Zersplitterung der slowenischen Sprache. Ebenfalls 1555 folgte Ena Molitov tih Kerffhzhenikou, dessen Autor Vergerius war, wobei Trubar als Übersetzer wirkte.

Daraufhin machte sich Trubar an die Übersetzung der übrigen Evangelien, so dass 1557-1558 der Erste Teil des Neuen Testaments veröffentlicht wurde (Ta pervi deil tiga Noviga Testamenta, vtim fo vsi shtyri evangelisti inv tv diane tih Iogrou, fdai peruizh vta Slouenski Iefik, Skufi Primosha Truberia fueiftu preobernen). Das Buch ist ein umfangreicher Sammelband, der neben den Evangelien eine deutsche Widmung, das umfangreiche slowenische Vorwort Ana dolga Predguuor (= P 1557/58) und eine Postille enthält.

1560 setzte Trubar sein Werk mit dem Zweiten Teil des Neuen Testaments fort (Ta drugi deil tiga Noviga Testamenta, vim bosh imel vse listy inv pisma tih Iogrou, Skratkimi inu Saftopnimi Islagami, Sdai Peruizh is mnogeterih Iefikou, vta Slouenski, skufi Primosha Truberia Crainza, fueiftu preobernen), und zwar erschien zunächst nur der Römerbrief ( $T a$ List h tim Rimljanom $=\mathrm{R}$ 1560). Er enthält die deutsche Widmung an den König Maximilian, in der Trubar über die Notwendigkeit des Dolmetschens spricht, da das einfache Volk keine andere Sprache als die slowenische verstehe. In demselben Jahr luden die Krainer Landstände Trubar ein, als Predi- 
ger in die Heimat zurückzukehren. Bevor er dies tat, organisierte er jedoch die Slawische Bibelanstalt in Urach bei Tübingen, deren Leitung er Hans Ungnad anvertraute. Über die Tätigkeit der Bibelanstalt besteht eine reichhaltige Literatur (grundlegend Bučar 1910, über Trubars Anteil Rotar 1988); sie arbeitete bis 1564. Aus Trubars deutschen Widmungen zu den slowenischen und kroatischen Büchern erfahren wir Vieles über seine Ansichten zur sprachlichen Situation auf der Balkanhalbinsel (alle Vorwörter gesammelt und nachgedruckt in Sakrausky 1989, verarbeitet in Katičić 1996). Die Übersetzertätigkeit werde die Türken zum rechten Glauben zurückführen. Im selben Jahr erhielt Trubar die Einladung der Krainer Landstände, als Prediger nach Laibach zurückzukehren, was dieser auch tat.

In der Zwischenzeit arbeitete Trubar weiter an den Episteln und veröffentlichte 1561 die Korintherbriefe und den Galaterbrief (Svetiga Pavla ta dva listy htim Corintariem inu ta htim Galateriem). 1562 erschien Articuli oli deili te prave stare vere kershzhanske (Drey Christliche Confessionen, namlich Augspurgische, Wirtembergische und Sächsische), eine freie Bearbeitung des Ausburger Bekenntnisses mit Ergänzungen (Rupel 1965: 164). Es folgte das Gesangsbuch Ene duhovne peisni (= DP 1563).

Mit der Einführung des Protestantismus in Krain wurde die Frage der Kirchenordnung aktuell. Da eine solche Kirchenordnung über theologische und kirchliche Angelegenheiten hinausging, wäre es Sache des Landesherrn gewesen, sie zu genehmigen. Dies kam jedoch nicht in Frage, weil der Landesherr ein eifriger Katholik war, und so veröffentlichte Trubar seine Cerkovna Ordninga 1564 (= CO 1564) ohne Genehmigung, was für Trubar die bekannten schwerwiegenden Folgen haben sollte. Er musste seine Heimat wieder verlassen, diesmal für immer.

In der weiteren Folge veröffentlichte Trubar seine Übersetzung der Psalmen (= Ps 1566) und 1577 die noch fehlenden Teile des Neuen Testaments (Noviga Testamenta pusledni deil). Das gesamte Neue Testament erschien 1582 als Buch in zwei Bänden. Auf die übrigen Werke soll hier nicht im Einzelnen hingewiesen werden; sie sind in Rupel 1965 (293-298) und Berčič 1968 (168-245) verzeichnet.

Unter den Nachfolgern Trubars seien besonders Sebastian Krelj und Jurij Dalmatin hervorgehoben, die Trubars Orthographie deutlich verbesserten. Eine wichtige Rolle spielte die Revisionskommission der Dalmatinschen Bibelübersetzung 1581, durch die Unzulänglichkeiten von Trubars Rechtschreibung beseitigt wurden. Sie wurde in Bohorič’ Grammatik 1584 kodifiziert (Ramovš 1971).

Wir werden im Folgenden einige Merkmale der Sprache Trubars behandeln, ohne dabei Anspruch auf Vollständigkeit zu erheben. ${ }^{1}$

${ }^{1}$ Ich danke meinen Studentinnen, die in meinen sprachwissenschaftlichen Seminaren Trubars Texte K 1555, M 1555, P 1557/58 und CO 1564 elektronisch aufbereitet haben, ebenso wie Frau Dr. Herta Maurer-Lausegger und ihren Studentinnen, in deren Seminar Dalmatins Vorrede 1584 eingegeben wurde, für ihre mühevolle Arbeit und die Überlassung der Daten. Die übrigen angeführten Texte habe ich selbst elektronisch bearbeitet. 


\section{ORTHOGRAPHIE UND PHONETIK}

\subsection{Schreibung des $l$}

In $\mathrm{K} 1550$ wird das velarisierte $l$ vielfach als $l l$ geschrieben, z. B. rekall, imenouall, ieidell (Neweklowsky 1984a: 141). Dort gibt er auch Anweisungen für die Aussprache des $<\mathrm{l}>$ (,In diefer vnferer Windischen fprach mueß du... das L zu zeiten grob auff Vngrisch oder Bisyackhisch... außzufprechen dich gewönen“) und im Abecedarium 1555 („LVbi Slouenci, Vom ie potreba fe nauuzhiti inu nauaditi,... zheftu inu ta L. zhafi debelu po Befiafhku ifrezhi“). In K 1555 ist diese Schreibweise nur noch in folgenden Wörtern zu finden: cillu ( 2 mal - aber 13 mal cilo, cilu), tellu (15 mal), oblazhillu (3 mal), dellu „Werk, Tat“ (24 mal, neben 4 mal delu), dazu dellati (3 mal in der Verbindung shpot della, neben mehrmals delati), dopallu (einmal), zoll (3 mal), wobei auffällt, dass es im Auslaut (Perfekt) nicht mehr vorkommt (Neweklowsky 2002: 242). Im Abecedarium 1555 ist im Auslaut kein $l l$ mehr vorhanden (aber tellu), in M 1555 nur in ganz wenigen Beispielen zwischen Vokalen (Ogris 1999: 93, führt dellu, tellu und uallue ,valove“ an). Trubar normalisiert relativ bald einfaches $l$ in allen Positionen mit Ausnahme einiger weniger Wörter, in denen es bleibt (dellu, tellu in P 1557/58, dazu kommen Poftilla und molli, in CO 1564: plazhillu, oprauillu, billu, billa, fgubilla, fazhella, kmallu, slushilla, dobilla, zheftillu, hualillu, veffellu, krall „König“, fterll). Es fällt auf, dass auch hier am häufigsten -llzwischen Vokalen steht.

In Bohorič' Grammatik 1584 wird auf die „dicke“ Aussprache des $l$ hingewiesen (Toporišič 1987: 294), es finden sich aber nur sehr wenige Beispiele, wo tatsächlich ll steht, z. B. Kardellu, Smolla.

\subsection{Die $s$ - und $\check{s}$-Laute}

Auch in den späteren Werken tritt $\iint$ für stimmloses $/ \mathrm{s} /$, gelegentlich auch mit oder ohne $h$ für $/ \check{s} /$ auf. Es scheint, dass Trubar schon eine Lösung für die Opposition $s: z$ mit den vorhandenen Buchstaben $\int$ und $s$ anstrebte. In den beiden ersten Werken mit Frakturschrift hängt die Schreibung „langes“ oder „rundes“ $s$ nicht von Trubar, sondern vom Setzer ab (Rigler 1968: 216 ff., Toporišič 1986: 272 f.). Einige Beispiele in Ps 1566: piffali, beffeda, Nebeffa, Veffelie, traditionell auch in groffovitu (= grozovito). Die Lösung war aber bei Trubar noch nicht gefunden.

Sie kam erst mit Sebastian Krelj (seit OB 1566 und seiner Postille 1567, die ich nicht einsehen konnte), und er schreibt $\int$ für $/ \mathrm{s} /$ und $s$ für $/ \mathrm{z} /$, analog für die Zischlaute: $\int h=\check{s}$ und $s h=\check{z}$ (Rigler 1968: 219 f.), während es für das unpaarige /č/ mit $z h$ nie Schwierigkeiten gegeben hatte. Bohorič führt in seiner Grammatik an, dass $\int$ das kroatische $<\mathrm{s}>$ wiedergebe, $s$ dagegen das kroatische $<\mathrm{z}>$, wobei diese Buchstaben in glagolitischer Schrift angeführt werden, also eindeutig sind (Toporišič 1987: 51).

Von allen Texten wurden Konkordanzen und Wortlisten, die die Auffindung der Daten wesentlich erleichterten, hergestellt. 
Diese Lösung geht allerdings auf Sebastian Krelj zurück (Toporišič 1966: 25), trägt aber Bohorič’ Namen (bohoričica).

Dalmatin hat die Unterscheidung der beiden Phoneme $s: z$ schon in seinen Werken vor der Bibelübersetzung durchgeführt, während die Unterscheidung $\check{s}$ : $\check{z}$ erst 1584 kam (Ramovš 1971: 151). Ein Beispiel aus Sirach 1575 (S. 3): Kadar fim ieft vshe vEgypsko deshelo bil prishel, vtim ofmim inu tridefsetim leitu, vtim zhafsu kraila Ptolemea Euergeta, inu fim bil ondu veliku zhafsa, fim ieft sadobil proftor k'ueliku dobrimu brainu inu pissainu. - In der Bibel wird das System schon sehr konsequent angewandt, nur bei der Großschreibung wird nicht zwischen stimmhaft und stimmlos unterschieden: es gibt nur einen Großbuchstaben S (Sakaj, Satu, Syn, na Semli, po vfem Svejti, Svetniki), sonst ferce, saftopnoft, fposnajo, od sazhetka, snami, resodeti, isvuna, s'uftmi (z ustmi). Wenn sich die Stimmbeteiligung automatisch (in phonologisch schwacher Position) ergibt, kann für stimmloses $/$ s/ auch $<$ s $>$ stehen: s'joim, s'Jhpendio (̌̌pendija „Nahrung“). Die Opposition $\check{s}: \check{z}$ (pregrefhnim, Jhtrajfa, prifhàl, pifhe : shlaht, dèrshali, Boshji) wird ebenfalls durch $\int$ und $s+\mathrm{h}$ unterschieden. Dennoch wird $\iint$ zwischen Vokalen oft beibehalten: sapiffati, Beffeda, $s u \iint e b, z h a \int f u$. Es handelt sich um eine Weiterentwicklung von Trubars System, wobei die Tradition bereits so stark war, dass die nunmehr unnötige Konvention $\left(\iint\right.$ in bestimmten Wörtern) beibehalten wurde. Abweichungen von diesem System kommen aber gelegentlich vor.

\subsection{Die Schreibung des Jat}

Die Distribution der Reflexe $e$ und $e i$ für das alte Jat hat Rigler ausführlich beschrieben (Rigler 1968: 32-82). Es überwiegt der Reflex $e$, während das Unterkrainer $e i$ in ganz bestimmten Positionen und Funktionen gebraucht wird (nach Logar 1996 dort, wo es auch im Oberkrainer Dialekt auftritt), und zwar zur Unterscheidung von Homonymen, ferner im Auslaut (von wo es in bestimmten Beispielen auch in andere Positionen übernommen werden kann) und vor dem Konsonanten $s$. Dieses System gilt von allem Anfang an. Dennoch hat sich das System entwickelt und zeigt in den jüngeren Werken größere Stabilität. In K 1550 finden wir: 29 mal fueidt „Welt“ gegen fuit „Rat“" (3 mal), fuet „,heilig“ (135 mal konsequent); von 17 Formen des Substantivs delo werden acht mit ei, neun mit $e, 16$ mit $l l$ und eine mit $l$ geschrieben, während von neun Formen des Verbs delati vier mit ei, fünf mit $e$, fünf mit $l l$ und vier mit $l$ geschrieben werden, so dass es durchaus zur Homographie einzelner Formen der Paradigmata von delu und delati - nämlich dela - kommen kann (Neweklowsky 1984: 142). Die Zahl der Schwankungen nimmt bereits in K 1555 ab. Hier finden wir elfmal deil ,Teil“", während die Formen von delu „Werk, Tat“ 24 mal mit dem Stamm dell- geschrieben werden (und nur einmal del-); delati wird zum Unterschied davon 9 mal dela- geschrieben, viermal allerdings della-. Während in diesem Beispiel 1550 noch ei und $e$ abwechseln, ist der Reflex des Jat 1555 als $e$ normiert und nur die $l$-Schreibung schwankt noch (vollständige Liste der Formen mit ei im Katechismus 1555 in Neweklowsky 2002: 244). Das System in M 1555 
entspricht dem im Wesentlichen ebenfalls (Ogris 1999: 36-42). In R 1560 werden die Formen von „essen“ 14 mal mit ei geschrieben (ieifti, ieido, ieidel, ieila), nur einmal mit $e$ (iesti); die Unterscheidung deil „Teil“ und delu, delati ist konsequent, Unterschiede gibt es aber immer noch bei der $l$-Schreibung (Neweklowsky 1995: 314 f.).

Beispiele aus CO 1564: deil „Teil“, obdeili, fueiftu, na tim Sueitu „Welt", hti Ieidi, po nega zhudnim, modrim fueitu „Rat“, Seime, leitu, imeiti, imei, imeila, poueidati, vmeili, vdoftih meiftih, veiditi / veideti, veidila, veim, vei, veifte, veimo, veido, veift, 3.Sg. dei, deimo, obei (duei) Naturi, utim fercei, usw. Folgende Schwankungen bei Jat (ei / e) werden für CO 1564 in Logar (1996: 352) angeführt: umetelen, človeški, človestvo, vest, zvest, svet, greh, mesto, nevesta, dete, bežati, povedati, imeti, smeti. Ps 1566: beishal, deil, deimo, imeili, na tim fueitu, leit, veiditi, veidezhe, veido.

In Bohorič' Grammatik gibt es einige Abweichungen: fejme / Seme, en dejl jejdi, vejm, vej, dejm, dvej, Mejftu, delati, delam, Iem, Iefti, Iedil; Svet „Welt“ (Svet -iga „heilig“, Svit „Rat“), (i)meti, povédati, Delu -la, Létú „Jahr“, Poléti, svesde, sveftu, Bei Bohorič kommt ei sparsamer vor als bei Trubar (die Beispiele können leicht in Stabéj 1971 aufgefunden werden).

Dalmatin schreibt im Vorwort zur Bibel 1584 ej in folgenden Beispielen: na tém Svejti , dejl „Teil“, refvejtil, rejs, vej, vejdo, vejdezh, vejden, vejdili, vejditi, mejftih, premejnil, Jvejzha, Jlejdni, frejdnik „Vermittler“, premejnili, mejnio, fo mejnili, lejt, polejtnimu, prejd, svejfzhino, fvejtil, rejs, vrejdnu, krejg „Streit“, vmejs, vejter, vejft, sveifti, dvej, obej, vrejden, vreidnoft, rejshezh, rejfhno kry „das erlösende Blut“, rejshezh Mezh, rejfhovati, namejftiti, vlejkli, v'fvoim meffei, fvejtio, refvejti „erleuchtet“, vrejzhi, dejmo, vmejs, s'svejsdizami, ftrejzhi, blejfhane. Bei Dalmatin erhält das betonte Jat häufig den Akut (é), so dass damit möglicherweise ej bezeichnet werden konnte (Ramovš 1971: 147). Freilich fällt auf, dass ej und é nicht schwanken (z. B. finden wir befféda, gréh, sapouédal, sapovém, iméti, némajo, v'téh Buqvah, u'vfeih, Lyftéh, lépu, tèrpéti; dazu kommen noch Beispiele, wo é nicht auf altem Jat steht (unten).

Aus den Beispielen kann ersehen werden, dass Trubars System im Prinzip auch von Dalmatin übernommen wird. So wie Trubar die Verwendung des Diphthongs $e j$ aus Gründen der Überregionalität einschränkt, glaubt er, dies auch mit anderen Vokalen machen zu müssen, z. B. finden wir hufhi für hujši, mlafi für mlajši, mahin für majhen (Rigler 1968: 28 f.). In K 1555 (und anderswo) rasbonik, u. a.

\subsection{Umlaut nach historisch weichen Konsonanten}

Nach weichen Konsonanten geht $-a$ in manchen Fällen, am häufigsten nach historisch weichem $r$, in -e über. Diese Erscheinung ist im Gen. (Akk.) Sg. der Maskulina und der Neutra anzutreffen, nicht aber im Nom. Sg. der $a$-Stämme, daher ist das Allomorph nicht nur phonetisch bedingt, sondern auch an bestimmte Deklinationsklassen gebunden. Außerdem kommt es beim Verb auf -ati vor. Neben den umge- 
lauteten Formen treten auch unveränderte Varianten auf. Beispiele in K 1550: hudizhe / hudizha; od primosa trubarie, in K 1555: od hudizhe, ferditiga Rihtarie (,Richter"), od tiga drukarie („Drucker“), nei obeniga Altarie kushoual; Neutra: obeniga resgledane, tiga giane, taciga faslushene, in M 1555: od Gofpodarie, tiga Vifhiga farie („Pfarrer“), deshelskega flegarie („Landespfleger“), eniga pridigarie, eniga lonzharie, tiga Ceffarie, aber paftiria / Paftyria, karneria; Neutra: ozhi fo pelne fpane (= spanja), is tiga preobrozhouane, od uoiskouane, aber Od farozhena Marie, Od ubyena, Od Vftanena, vashiga gouorena, in P 1557/58: od Hudizhe (3 mal), Sludie (4 mal), Pridigarie (3mal), tiga Tolmazharie; nega diane inu faslushene (mehrmals), tiga Tolmazhene, od celiga Ohranene inu fuelizhane, Is letiga premishlene, fa volo tiga dershane inu ohranene, aber ferdamnena, Goriuftanena, pres hudiga perklonena, gouoriena, tiga veidena usw. In CO 1564 finden wir: od fuiga Pridigarie, beim Neutrum auf -anje, -enje häufig - $e$ : od falsh neuerniga klyzane inu Molene, dobriga diane, polni zbiulane, pres nafhiga faslushene, od Odpushzhene, tiga Terplene, usw. Logar (1996: 354) führt für CO 1564 die Verbalformen preganaio / naganeio, poshila / pofhile (= pošilja) an. In Ps 1566 finden wir: preganeio, vftaieio, aber opominaio.

Jedenfalls ist der Umlaut ( $e<a$ ) ein Unterkrainer Merkmal, während in den Oberkrainer Dialekten das $a$ bleibt (Logar, loc. cit.).

Die ersten Verbesserungsvorschläge zu Trubars Orthographie kamen vielleicht von den Protestanten, die 1563 Ene duhovne peisni herausgaben (so drückt sich Toporišič 1992: 241 f., aus), und neben der Beseitigung des Umlauts eine Reihe von weiteren Verbesserungen einführten.

Die umgelauteten Beispiele nach weichem Konsonanten nehmen aber auch bei Trubar kontinuierlich ab; die Normierung geht in Richtung zur Endung $-a$, das bei Dalmatin und Bohorič 1584 die alleinige Endung ist.

\subsection{Die Endung -om im Dativ Plural der $a$-Stämme}

In K 1550 finden wir folgende Formen der $a$-Stämme: besedom, shenom, sirotom, vduuom (Übergang von $a$ in $o$ vor $-m$, außerdem in vom, nom / nam). Man kann die Hebung des $a$ zu $o$ vor nasalen Sonanten als Teilassimilation erklären (sie kommt in vielen Sprachen vor): Beim nasalen Labial ist die Mundhöhle geschlossen, der vorhergehende Vokal wird gehoben und damit die Schließung vorbereitet. Wir gehen davon aus, dass es sich (wegen nom, vom) um ein phonetisches Gesetz handelte und nicht um die Übernahme der Endungen der $o$-Deklination. Beispiele: in K 1555 moshem inu shenom, Le-tim beffedom, knom pride, tym Syrotom, pomagaite tim Vduuom, tim Viudom; Maskulina (wie erwartet): tim nebeskim erbom, htim Iudom, dazu vom (Endung -am kommt nicht vor); in M 1555: knogom, pokoi vashim dushom, shenom, nom, vom, kuom, quom (kein -am); htim hlapcem, kopazhem, vom Pifariem inu Farifeiom, Iogrom, muim bratom, slufhabnikom, nashim dolshnikom, otrokom; in P 1557/58: tim oblubom, muim beffedom, nadlugom, nih beffedom Pridigom inu pifmom Verouati, nom, uom, vom; Tim Sludiem, pokorni Gofpodariem inu 
Gofpodinom, Pridigariem, Galatariem, Sapuuidom, Vudom, Malikom; in CO 1564: Faryem Menihom inu Nunom, oblubom, nadlugom, htim ferzom, nom neben nam (mehrmals), uom, tim mladim Otrozhizhem, htim Rihtariem, Kralem.

In der Grammatik Bohorič' finden wir -am normiert (materam, S. 55 bzw. Toporišič 1987: 79, pifmam, S. 60 bzw. Toporišič 1987: 84), ebenso schreibt Dalmatin 1584: beffedam, sapuvidam, nogam, nadlugam, gmajnam, Sirotam, Buqvam, Vduvam, Svetnizam, ebenso bei den Neutra: dellam, Nebeffam; plus nam, während die Mask. die erwartete Endung -om /-em besitzen: Rihtarjem, Ebreerjem, Paftirjem, k'Iudom, h'gréhom, Erbizhom, Hudizhom, zhlovekom, vfem Slovenom; ähnlich sind schon die Formen in Moses 1577.

\subsection{Die Sonanten $l j, n j$}

Die Sonanten $l j n j$ werden zunächst nicht unterschieden, z. B. M 1555: Gen. Sg. femle, aber vefelie, olia, olie in einzelnen Wörtern. Mit R 1560 wird die Bezeichnung des palatalen $l j$, allerdings nur im Wort veselje, normiert: Veffelie, Ueffelie, Ueffelia, Vesselia, Ueffeliem, Veffeliom (vgl. Toporišič1986: 274). In CO 1564 finden wir im Gen. Pl. einige Male $-y$ (= ji), z. B. shely (fo polne hudih shely). Logar (1996: 355) gibt für CO 1564 an, dass die Schreibung am häufigsten $l n$ ist, aber die Palatalität doch öfter gekennzeichnet wird: faflushenie, terplenie, fanne / Saine / faine (za nje)...; semlio, uolio, fueffeliem, vola, krali...

In DP 1563 werden $l j n j$ gewöhnlich von $l n$ unterschieden (Toporišič 1992: 242).

Dalmatin hat sich vor der Tätigkeit der Reformkommission 1581 an Trubars System gehalten (häufiger aber in, $i l$ als bei Trubar; Sirach 1575: gouoriene, premagai$n e, n y u=n j i j u$, Ogen, sa nih voilo, po... sheilah, Semla, ludy, in die Bibel wird aber $n j$, seltener $j n$ oder $n$ eingeführt; Ähnliches gilt für $l j$, für das auch $j l$ oder $l$ geschrieben werden, jedoch deutlich inkonsequenter als bei $n j$ (Ramovš 1971: 148).

\subsection{Schwankung bei den Präpositionen bzw. Präfixen $n a, z a, n a d, r a z$}

Uneinheitlichkeit herrscht bei $n a, z a, n a d, r a z$; bei den ersten drei finden wir im Unterkrainer Dialekt vor Vordervokalen die Assimilationsformen ne ze ned, während raz immer zu rez wird. Schwankungen finden sich nach diesem Modell, allerdings selten, z. B. nafe vfel / nefe ufel (= na / ne se vzel), na konzu / ne konzu, fauupamo / Seuupamo (Logar 1996, 354). In K 1555 finden wir einmal ne zhaft, in M 1555 ned ti pocuri, ne Sepela, fepufti / Japufti, in P 1557/58 neunmal Formen von fepelati, sonst $\int a-$.

Dalmatin hat rez konsequent übernommen: refodil, resdilenih, reslozhiti, resmiflimo; keine Beispiele mit ze: sahvalimo, sakrivam, samaknen, samerkati, samolzhal, sanefti, savupati, keine mit ned, ne (na). Die umgelauteten Formen sind schon bei Trubar selten, Dalmatin hat die Oberkrainer Formen mit Ausnahme von rez normiert. 


\subsection{Weitere Neuerungen}

Trubar verwendet keine Akzentzeichen, sondern zur Bezeichnung der Vokallänge werden des öfteren Doppelbuchstaben gebraucht. Akzentzeichen werden zuerst von Krelj in der Postille 1567 verwendet, und dann von der Revisionskommission eingeführt (Ramovš 1971: 157), und zwar Akut zur Bezeichnung der betonten Länge (Beispiele auf Jat s. oben, öfter zur Kennzeichnung der betonten Kurzformen in der 3. P. Pl.: pokoré, dèrshé, vuzhé, sapufté, beró, tekó, raftó, zvetó, isrekó, andere: is ... gláve, od Jvetiga Duhá, praviga vuká, Bogá, Serzá, dán, nesná, nevpelá, moriti, nóssi, njó, na Púli, selten bezeichnet á die betonte Kürze: bilá, gelegentlich kommen auch Druckfehler vor: sa kateriga voló). Der Gravis dient zur Bezeichnung des silbischen $r$ und des Schwalauts: pèrviga, potèrdi, pèr tebi, Kàrfhzhanfke, kakòr, mogèl, rekàl, ni/htèr; er ist nicht an die Akzentstelle gebunden.

Die Verbindung der unsilbischen Präpositionen mit ihrem Beziehungswort wird schon in Sirach 1575 eingeführt s'shaloftnim, v'ueffeile, k'mafzhouaine, und später von der Reformkommission in die Bibel und von Bohorič in seine Grammatik übernommen (vgl. Ramovš 1971: 154).

Dalmatin kommt auch das Verdienst zu, die beiden Varianten des lateinischen $V$, $u$ und $v$, zur Lautunterscheidung eingeführt zu haben, noch vor 1581.

\section{MORPHOLOGIE}

3.1 Die Kurzformen der Adjektive und andere Besonderheiten

Trubars Katechismus von 1550 ist sprachlich das archaischste seiner Werke und von daher eine wahre Fundgrube für die historische Grammatik des Slowenischen (Rigler 1968: 11, Anm. 1). Hier sei zuerst auf die Kurzformen der Adjektive hingewiesen, die von ihrer Form her interessant sind, aber bei Trubar nicht mehr die Funktion „nichtdeterminert“ besitzen. Diese Unterscheidung übernimmt der bestimmte und unbestimmte Artikel. Es handelt sich um folgende Formen: le ta zhuden fuit boJhy, ${ }^{2}$ le ta fkriuen zhuden fuit, Ta prauizhin bode is fuye Vere Jhiu, ta velik ferd boShy, ta velik tadel, tui velik ferd, vtim flouenski iefiki se praui, vtim erdezhu moryei, na taki dobri oblubi inu faguifhanim bofhyei, vgl. auch: vdobrim tar vflei (mit dem Reim 'naprei'). Solche Formen können wir auch noch später finden, allerdings in geringerer Anzahl. Beispiele aus P 1557/58: ta nash velik Trosht, ta preuelik ferd Boshy, ta faslushen lon, ta uelik dar Boshy, ta zhuden, moder inu skriuen Suit. Trubar normiert im Laufe der Zeit die Langformen.

Eine Besonderheit ist die Dativform des Adjektivs dober, und zwar hdobrumu „zum Guten“ und Ta isti, tiga Verniga, serze, misal inu vse giane premeni vsimu dobrumu. Phonetisch ist dies eine Assimilationserscheinung, die freilich nur in dem ei-

${ }^{2}$ Die Beispiele für K 1550 können in Neweklowsky 1984b lokalisiert werden. Sie beziehen sich auf den eigentlichen Katechismus. 
nem Wort vorkommt. Bereits in K 1555 und in P 1557/58 kommt diese Form nicht mehr vor, sondern nur noch hdobrimu.

\subsection{Der bestimmte Artikel}

Die Einführung des bestimmten Artikels ( $t a, t a, t u$ ) erfolgt nach deutschem Vorbild beim Übersetzen; davon zeugt Kap. Vera in CO $(1973,101)$, das schon vor ihm übersetzt und verwendet wurde (Logar 1996, 352). Einen Überblick über die Formen des Artikels in K 1550 findet man in Neweklowsky 1984b (278-305).

Dass Dalmatin mit dem Artikel sparsam umgeht, sehen wir schon am Beginn von Moses 1577: Koku ie Gofpud Bug vsheftih dneh vfe Stuari, Nebu, Semlo, Sonce, Luno, Suesde, Trauo, Lyftie, inu vfakershne Suirine, katere V'uodi, Vluffti, inu na Semli shiueio, puslednimu tudi Zhloueika Jtuaril.

\subsection{Die Paradigmen des Typs bog und srce}

Auf Interesse stößt das heute bewegliche Paradigma des Typs bog oder srce. Die Beispiele aus K 1550, aber auch später, belegen, dass sich dieser Akzenttyp gerade zu Trubars Zeit herausgebildet hat. Dies kann man vor allem mit Hilfe der Vokalalternation $o$ (unbetont) : $u$ (zirkumflektiert) feststellen (Neweklowsky 1986). Die Beispiele zeigen, dass im alleinstehenden Wort oder wenn diesem ein anderes akzentuierbares Wort vorausgeht, im Singular die Endung betont war, entsprechend dem slowenischen Akzentverschiebungsgesetz, dass der urslawische Zirkumflex auf die folgende Silbe verlagert wird (dem entspricht Stammakzent im Russischen und fallender Stammakzent im Serbokroatischen, russ. bog, bóga; skr. bôg, böga). Befindet sich das betreffende Wort jedoch in unmittelbarer Nachbarschaft einer Präposition, wurde der Akzent auf den Stamm zurückgezogen (im modernen Slowenischen nur in Resten (z. B. poljê : na pólje).

\section{K 1550}

$\begin{array}{lllll}\text { Nom.Sg. } & & & \text { Bug } & \text { (208 mal) } \\ \text { Gen.Akk. } & \text { Boga } & (61 \mathrm{mal}) & \text { v, od, na Buga } & (35 \mathrm{mal}) \\ \text { Dat. } & \text { Bogu, hbogu } & (83 \mathrm{mal}) & \text { hbugi } & (1 \mathrm{mal}) \\ \text { Instr. } & \text { *Bogum } & & \text { pred, z, vmei Bugom (51 mal) } \\ \text { Lok. } & \text { Bogu } & (1 \mathrm{mal}) & \text { per, na, po Bugi } & (13 \mathrm{mal}) \\ \text { Nom.Pl. } & \text { boguui } & (1 \mathrm{mal}) & & \\ \text { Gen. } & \text { bogou } & (2 \mathrm{mal}) & \end{array}$




$\begin{array}{lllll}\text { Nom.Sg. } & \text { fyn } & (42) & & \\ \text { Gen.Akk. } & \text { fynu } & (61) & \text { fyna } & \text { (1) } \\ \text { Dat. } & \text { finuui } & (4) & \text { fynu } & \text { (2) } \\ \text { Instr. } & \text { fynum } & (3) & \text { f fynom (1) } \\ \text { Dat.Pl. } & \text { fynuuom (1) } & & \end{array}$

Lok.Sg. $\quad$ vtim ferzei (7) vferzu (3), vferzi (3)

\section{K 1555}
Nom.Sg. bug
Gen.Akk. boga, v, od, na buga
Dat. $\quad$ g fserci, famimu Synuui
Instr. $\quad \mathrm{s}$ bugom, pred bugom, ftrahom, per ... Synom (!), pred obenim ftrahum, fred fuetim Duhum, fteim slatum
Lok. $\quad$ v muim fercei, u tim meflei, per bugi

Die Pluralendungen kommen selten, bei vielen Substantiven überhaupt nicht vor; die Stammerweiterung $u v$ war betont: boguui (= bogûvi). Der heutige bewegliche Akzent dieses Paradigmas ist durch eine Vermischung der beiden Paradigmen, mit und ohne Proklitik, zustande gekommen.

Die Endung -uvi im Dativ des Paradigmas von sin lautet synuиi (= sinûvi), daneben kommt auch die jüngere Form synu vor. In K 1550 tritt synuui viermal auf, einmal auch im Lok. Sg. Diese Form ist auch in K 1555 (einmal auf S. 129) und in der Langen Vorrede (elfmal) anzutreffen. In CO 1564 finden wir: per Boshym Synuui. In fteim Synum ist die Endung betont.

In CO 1564 finden wir in Übereinstimmung mit obigem System: od prauiga Boga : na Buga, slushio Bogu : Hbugi, sleteim Bogum : pred Bugom, ebenso in Ps 1566: od famiga Boga, D. Sg. Bogu, per famimu Bogu : Bug, na Buga, pred Bugom, Sbugom, per Bugi.

Die Alternation $o: u$ wurde teilweise in DP 1563 beseitigt (Duhom, nicht Duhum) (Toporišič 1992: 242), was aber keine Vorbildwirkung besaß, denn in der Bibel 1584 sind die Alternationen ebenfalls anzutreffen, z. B. s'bugom [= z bûgom] Ozhetom, Synom $[=$ s sînom $]$ inu S. Duhum $[=d u h u \hat{m}]$, aber s'fvoim Duhom $[=d \hat{u}-$ 
hom], pred Bugom (oft), einmal aber pred Bogom, nad letim vukum [= vukûm], pod Nebom, usw.

Der Lokativ dreisilbiger Neutra des Typs in K 1550 vposhtenei, vveselei und in $\mathrm{K} 1555$ vtim nebeskim vefelei, v poshtenei scheint später nicht mehr vorzukommen. In der slowenischen Standardsprache ist dieser Akzenttyp nicht mehr anzutreffen (dialektal ist er allerdings bekannt), der offene Vokal $e$ mit steigender Intonation weist jedoch auf alte Endungsbetonung hin. Der Typ hat seine Parallele im čakavischen Dialekt. In M 1555 fueffelem lässt sich nicht entscheiden, wo der Akzent lag, in CO 1564 heißt es des öfteren: bruma inu poshtene, veffelie (der Lokativ scheint nicht vorzukommen).

Die Varianten $-e i /-i /-u$ im Lokativ Sg. einiger Maskulina und Neutra verwiesen ebenfalls auf den Gegensatz betont ( $e i$, aus Jat) und unbetont ( $-i$ aus unbetontem Jat, $-u$ aus den $u$-Stämmen oder dem Dativ, nach Logar aus $-\ddot{u}$, s. unten), z. B. vtim serzei (srcêi) / vserzi (vsîci), vserzu (vŝ́cu). In K 1555 finden wir das Allomorph -ei auch bei den Substantiva duh und blago: vtim fuetim Duhei, vtim Duhei, gegenüber vtim duhu, na nih blagei, na nega zhafti, blagei inu shiuotu, u tim meffei (2 mal); in M 1555: vtuim okei; in P 1557/58 kommt duhei nicht vor, nur meffei, moriei und fercei, in CO 1564 blagei und fercei (vnega fercei : Vferci) ${ }^{3}$. Die Zahl der betroffenen Substantive nimmt kontinuierlich ab; wir können eine Normierung in Richtung Betonungsverlust und Ausgleich mit der Dativendung $-u$ beobachten.

Nach Logar (1996: 352) handelt es sich jedoch um eine phonetische Erscheinung. Trubar habe aus überregionalen Gründen sein dialektales $\ddot{u}$ aufgegeben, wovon ,Zweifellos“ die Schreibweisen kserci, hbugi, kmali mit der Endung $i$, die leicht vom $\ddot{u}$ erklärlich sei, zeugen. Ramovš’ Meinung, dass es sich um Übernahme der Lokativendung handle (1952: 39) scheint Logar unannehmbar.

Die Alternation $e: i$ im Stamm kommt selten vor, z. B. in rezh, „Sache“ : rizhi / rizhy, rizhio, rizhmi, rizheh (endungsbetont). In K 1555, M 1555 und P 1557/58 steht kein $y$ in der Endung, während es in CO 1564 häufiger vorkommt.

\subsection{Die Häufigkeit des Gebrauchs der Kasus in CO $1564^{4}$}

Roblek (2000) hat die Häufigkeit der Kasus und Deklinationstypen beim Substantiv in CO 1564 ausgezählt. Sie kommt dabei zu folgender relativer Verteilung: 1. Nominativ 32\%, 2. Akkusativ 25, 3. Genitiv 19, 4. Lokativ 13, 5. Dativ 6, 6. Instrumental 5\% der Fälle. Diese Verteilung lässt sich mit der Verteilung der Kasus in der modernen Sprache durchaus vergleichen.

Die Häufigkeit der Deklinationstypen sieht folgendermaßen aus: 51\% Maskulina, 26\% $a$-Stämme (meist Feminina), 15\% Neutra, 8\% Feminina auf -i. Eine solche Verteilung entspricht nicht den Durchschnittswerten in der modernen Sprache. ${ }^{5}$ Die

\footnotetext{
${ }^{3}$ Es wurde nur rund ein Drittel der Kirchenordnung ausgewertet.

${ }^{4}$ Nach Katerina Roblek, Seminararbeit 2000.
} 
große Zahl der Maskulina ist durch die Häufigkeit maskuliner Substantive wie Bug, Sin, Duh, greh sowie der Eigennamen bedingt.

Die Deklination der $a$-Stämme entspricht dem modernen Typ (abgesehen von der Endung des Dat. P1., s. oben); Schwankungen bestehen beim Typ auf -ev, wo es in CO 1564 im Instr. Sg. fto nega Molytuo heißt. Der Gen. Sg. von odrešitev lautet od tiga Odreshitua.

Im Gen. Pl. der $i$-Stämme kommt neben der regulären Endung $-i$ auch die Nullendung (Ramovš 1952: 63) vor: In CO 1564 heißt es: is tih Sapuuidi / tih Boshyh Sapuuid (ebenso K 1555). Andererseits dringt die Endung $-i$ auch in die $a$-Stämme ein: od nefpodobnih miffal, sheli. Daneben finden wir auch -ih: od ftuarih / ftuary. In zapoved lautet der Dat. Pl. auch -om nach der a-Deklination: Sapuuidom, pruti takim mislom, ebenso -ah (K 1555 po Boshyh Sapuuidah), -ami (Sapuuidami, P 1557/58, CO 1564). Die $a$-Deklination beeinflusst die $i$-Stämme auf verschiedene Weise, z. B. Akk. Sg. tuio mislo (M 1555).

Die Deklination von kri besitzt nur die Formen kry und Instr. kryo.

Nach Roblek (2000) kommt bei den Neutra zu 79\% die Endung -e und zu 21\% die Endung $-u$ beim Neutrum vor. Die häufige Endung - $e$ geht auf Konto der Verbalsubstantive. Über die umgelautete Endung - $e$ im Gen. Sg. wurde schon oben gesprochen. Auffallend ist slega (zlega), wovon oben ebenfalls gesprochen wurde. In seltenen Fällen wird im Dat. Sg. die Endung -i gebraucht (kferzi wie Hbugi) (s. oben, vgl. Ramovš 1952: 39). Im Lok. Sg. finden wir $-u$ neben $-i$, betont $-e i$. Nach der Zählung von Roblek entfallen bei den unbetonten Endungen auf - $u 67 \%$ und auf -i $33 \%$, während auf das betonte -ei $14 \%$ aller Belege kommen. Im Gen. Pl. kommt selten -ih vor (wie schon bei den $a$-Stämmen erwähnt). Im Lok. Pl. steht gewöhnlich -ih wie beim Maskulinum (M 1555: delih, meiftih, nebefih).

$\mathrm{Zu}$ den mask. $o$-Stämmen sei noch Folgendes gesagt: Das Lexem gospud hat in allen Kasus außer im Inst. Sg. die Endung -i. Ansonsten ist noch der Lok. Sg. zu erwähnen, wo wir wie schon beim Neutrum angedeutet, die Endungen $-u /-i /-e i$ (betont) finden. Letztere ist selten (M 1555: vtim duhei, CO: vtim mozhei).

Im Instr. Sg. und im Dat. Pl. und Du. konkurrieren nach palatalen Konsonanten und -c- die Endungen -em und -om bzw. -ema und -oma (Ramovš 1952: 42, 46 f., 51 f.). M 1555: fuefelom „mit Freude“, fcelim ferzom, pruti tim farifeiom, Slepzoma, fognom, Biryzhom D. Pl., tim Saduceiom : Steim preobrazhenem, fteim vupanem, gianem, sludiem, fteim dotikanem, truim uprashanem, Pifariem D. Pl., ognem, Sludeiem, fteimi sludiemi, fanem korcem, gfuim touarishem, le tim faryem, fuefelem, kopazhem D. Pl., hlapcem, fcelim tuiem fercem, fteim naprei daienem, htim Menaucem, pred... flegariem, ... plashzhem, tim Sholneriem.

Der Akk. Pl. der Maskulina lautet selten auf - $i$ aus (kosi, listi, voli, tati, gospudi, Logar 1996: 356). Hier handelt es sich wohl um einen Archaismus, der seine Entsprechung im Čakavischen besitzt. 


\subsection{Einige Verbalformen}

Beim Verb finden wir in K 1550 in drei Wörtern die alte Endung -o für die erste Person Sg. Präsens, und zwar veryo (8-mal), hozho (16-mal), nezho (3-mal). (In P 1557/58 kommen hozho und nezho vor, in CO 1564 ebenfalls hozho und nezho, dazu noch veruio). Eine größere Anzahl von Verben mit dem Bindemorphem - $i$ - hat in der 3. Person Pl. die Endung -e, z. B. sadobe (zadobiti), prosse / prossyo, posabe (pozabiti), gouore, daneben auch hote. Bei den Verben der $e$-Klasse erscheint öfters die Endung -o (neben gewöhnlich -ijo), z. B. prydo / prydeio, mogo (3-krat), fmoreio, nesso (nesti), odpro (odpreti) usw. (K 1550), in CO 1564: narede inu dershe, rede inu vuzhe, moree, dobee, dile, gouore, Jture; nefo, odpahno, pado, pregano, preoberno.

In Dalmatins Predgovor 1584 kommt nezhem einmal vor, nicht hozho, nezho, zweimal das formalhafte Ieft veruio. Beispiele für die verbalen Kurzformen s. oben 2.8. Bohorič hat Hozhem hoteti (S. 129, Toporišič 1987: 153), Nezhem vel nozhem nehtéti (130 bzw. 154), Verujem vel verjem, verúvati vel verjeti (133 bzw. 157).

\section{SCHLUSSFOLGERUNGEN}

Die Grundlagen der slowenischen Orthographie wurden 1550 von Trubar gelegt und sie bleiben mit gewissen Verbesserungen bis in die vierziger Jahre des 19. Jahrhunderts erhalten. Die Frage der Oppositionen $s: z, \check{s}: \check{z}$ wurde bei Trubar nur teilweise gelöst, dann von Sebastian Krelj verbessert, in Dalmatins Bibel bereits ziemlich konsequent durchgeführt und von Adam Bohorič in seiner Grammatik 1584 normalisiert. Bohorič und Dalmatin standen miteinander in Kontakt, denn ersterer war Leiter der Revisionskommission 1581 für die Bibelübersetzung, die ja schon 1580 fertig war (1584 gedruckt). Die Reformkommission führte den Buchstaben $j$ ein, den Gebrauch der Akzentzeichen, die Beschränkung des Gebrauchs der Großbuchstaben und des bestimmten Artikels; sie festigte ferner das $u$ als Resultat des Unterkrainer zirkumflektierten $\hat{o}$ (nuzh). Die Autoren der DP 1563 und Krelj OB 1566 haben sich nicht durchgesetzt. Die durch die Kommission eingeführten Änderungen sind im Vergleich der Bibelübersetzung mit früheren Werken Dalmatins zu erkennen; eigentlich ist dabei nur das $j$ wirklich neu, während die übrigen Verbesserungen schon bei Krelj und DP 1563 zu finden sind (Ramovš 1971: 131, Toporišič 1992).

Die $s$-Schreibung wird reformiert, doch bleibt $\iint$ in einer Reihe von Wörtern bewahrt, ebenso wie Trubars Schreibung des Jat. Bei der $l$-Schreibung hat sich die Schreibung $l l$ in gewissen Wörtern gehalten; Bohorič hat fast alle Wörter mit einfachem $l$, aber bei Dalmatin ist die Trubarsche Tradition in manchen Wörtern beibehalten. Gewisse Unterkrainer Elemente wie Assimilationserscheinungen bei Präpositionen (ne, ned, ze) werden ausgemerzt und nach Oberkrainer Vorbild normiert.

In der Deklination wird beim zirkumflektierten Paradigma das Unterkrainer $-i$ im Lok. Sg. durch das Oberkrainer $-u$ ersetzt, im Dat. Pl. der Feminina und Neutra wird 
-am normiert. Die nach palatalem Konsonanten umgelauteten Formen $(a>e)$ bekommen durchwegs $a$.

\section{Primärliteratur}

Bohorizh, Adam (1584): Arcticae horulae succisivae, de latinocarniolana literatura, ad latinae linguae analogiam accomodata, ... Witebergae 1584 [vgl. unten Toporišič 1987]

Truber, Primus:

K 1550

Abecedarium 1555

$=$ Catechismus in der Windischenn Sprach... Nachdrucke: Akademska založba, Ljubljana 1935, und Mladinska knjiga, Ljubljana 1970

$=$ Abecedarium. Ene buquice, is katerih fe ti mladi inu preprofti Slouenci, mogo lahku tar hitru brati inu piffati nauuzhiti. MDLV. Spremna beseda M. Rupel. München, brez leta (Südostverlag, Trofenik)

K 1555

$=$ Catechismus Vslouenskim Ieliku fano kratko fastopno Islago. 1555. Spremna beseda M. Glavan. Ljubljana 1992 (Državna založba Slovenije)

M $1555=$ Ta Evangeli svetiga Mathevsha. MDLV. Nachdruck: Spremna beseda M. Glavan. Ljubljana 1993 (Državna založba Slovenije)

P $1557 / 58$

R 1560

= Ana dolga Predguuor, s. Rajhman 1986

$=$ Ta drugi deil tiga Noviga Testamenta, vtim bosh imel vse listy inv pisma tih Iogrou, Skratkimi inu Saftopnimi Islagami, Sdai Peruizh is mnogeterih Iefikou, vta Slouenski, skufi Primosha Truberia Crainza, fueiftu preobernen (Tübingen 1560) [= Römerbrief, verwendet wurde das Exemplar des Instituts für Slawistik der Universität Wien, vgl. Berčič 1968, 178]

CO 1564

= Cerkovna Ordninga. Slowenische Kirchenordnung. Tübingen 1564 [Nachdruck München 1973: Trofenik]

Ps 1566

DP 1563

$=$ Ta celi Psalter Dauidou... / Der gantz Psalter in die Windische Sprach zum ersten mal verdolmetscht... Tübingen 1566

$=$ ENE DUHOVNE PEISNI, KATERE SO SKUSI PRIMOSHA TRVBERIA VTA flauenski yelik iftolmazhene... Geistliche Lieder in der Windischen Sprach... Tübingen 1563

Krelj, Sebastian:

OB 1566

= OTROZHIA BIBLIA / Ein Handtbüchlein, Darinn ist vnter anderm der Catechifmus Von fünfferlei fprachen. (Augsburg) [Nachdruck Ljubljana 1987 (= Monumenta litterarum slovenicarum 20 )]

Dalmatin, Jurij

Sirach 1575

$=$ JESVS SIRACH ALI NEGOVE BVquice (Latinski ECCLESIASTICUS)... vflovenski Iesik ftolmazhene / Jefus Syrach Windisch... Ljubljana [Nachdruck Ljubljana 1974 (Monumenta litterarum slovenicarum 12)]

Moses $1577=$ BIBLIE, TV IE, VSIGA SVETIGA PISMA PERVI DEIL, VKATERIM SO TE PET MOSESSOVE BVQVE, ... / Die Fünff Bücher Mofis... Ljubljana

Biblia $1584=$ BIBLIA, TV IE; VSE SVETV PISMV; STARIGA inu Noviga Teftamenta, Slovenfki, tolmazhena / Bibel, das ift, die gantze heilige Schrifft, Windisch. Wittemberg 
Berčič 1968:

Bučar 1910:

Katičić 1996:

Logar, 1996:

Neweklowsky 1984a: Gerhard Neweklowsky, Trubars Katechismus von 1550. - Eine Kon-

Branko Berčič, Das slowenische Wort in den Drucken des 16. Jahrhunderts, in: Abhandlungen über die slowenische Reformation, Redaktion B. Berčič (= Geschichte, Kultur und Geisteswelt der Slowenen 1), München, 152-268 + Abb.

Bučar Franjo, Povijest hrvatske protestantske književnosti za reformacije, Zagreb

Radoslav Katičić, Ein Ausblick auf die slawischsprachige Völkerwelt im Südosten (= Schriften der Balkan-Kommission, Philologische Abteilung 37), Wien

Tine Logar, Glasoslovne in oblikoslovne variante v jeziku Trubarjeve Cerkovne ordninge, in: T. Logar, Dialektološke in jezikovnozgodovinske razprave, uredila Karmen Kenda-Jež, Ljubljana, 351-356 kordanz, in: Protestantismus bei den Slowenen/Protestantizem pri Slovencih (= Wiener slawistischer Almanach, Sonderband 13), Wien, 133-152

Neweklowsky 1984b: Gerhard Neweklowsky, Trubarjev Katekizem 1550. Konkordanca, indeks besed, pogostnostni spiski, Ljubljana

Neweklowsky 1985: Gerhard Neweklowsky, Sprachliche Differenzierung innerhalb Trubars Katechismus von 1550, Slavistična revija 33, 153-161

Neweklowsky 1986: Gerhard Neweklowsky, Zur Paradigmatik in Trubars Katechismus 1550, in: 16. stoletje v slovenskem jeziku, književnosti in kulturi, uredila B. Pogorelec s sodelovanjem J. Koruze (= Obdobja 6), Ljubljana, 307-317

Neweklowsky 1995: Gerhard Neweklowsky, Die Entwicklung der slowenischen Schriftsprache in den ersten zehn Jahren 1550-1560. (Zum zweiten Teil des Neuen Testaments), in: Ein Leben zwischen Laibach und Tübingen. Primus Truber und seine Zeit, herausgegeben von Rolf-Dieter Kluge, München, 309-321

Neweklowsky 1999: Gerhard Neweklowsky, Razvoj slovenskega knjižnega jezika v prvih desetih letih 1550-1560, in: Logarjev zbornik: referati s 1. mednarodnega dialektološkega simpozija $\mathrm{v}$ Mariboru, eds. Zinka Zorko in Mihaela Koletnik, Maribor, 357-371

Neweklowsky 2002: Gerhard Neweklowsky, O jeziku Trubarjevega Katekizma 1555, in: Med dijalektologijo in zgodovino slovenskega jezika. Ob življenskem in strokovnem jubileju prof. dr. Martine Orožen, uredili M. Jesenšek, B. Rajh, Z. Zorko (= Zora 18), Maribor, 241-249

Ogris 1999:

Rajhman 1986:

Ramovš 1952:

Ramovš 1971:

Rigler 1968:

Rigler 1986:

Roblek 2000:

Rotar 1988:

Rupel 1965:
Martina Janja Ogris, Die Phonologie und Orthographie in Trubers Matthäusevangelium von 1555, Diplomarbeit, Klagenfurt

Jože Rajhman, Trubarjeva Ena dolga predguvor, Ljubljana

Fran Ramovš, Morfologija slovenskega jezika, Ljubljana

Fran Ramovš, Delo revizije za Dalmatinovo biblijo, in: Zbrano delo, knj. 1, Ljubljana (= SAZU, Dela 23/I, Institut za slovenski jezik 11/I), 140-152 [zuerst 1918 erschienen]

Jakob Rigler, Začetki slovenskega knjižnega jezika (SAZU, Dela 22, Institut za slovenski jezik 10), Ljubljana

Jakob Rigler, Razprave o slovenskem jeziku, izbral in uredil F. Jakopin, Ljubljana

Roblek Katharina, Besonderheiten in der Morphologie in Trubars Kirchenordnung [Seminararbeit, Manuskript], Klagenfurt

Janez Rotar, Trubar in Južni Slovani, Ljubljana

Mirko Rupel, Primus Truber. Leben und Werk des slowenischen Reformators, Übersetzung und Bearbeitung von B. Saria, München 
Sakrausky 1989: $\quad$ Oskar Sakrausky, Deutsche Vorreden zum slowenischen und kroatischen Reformationswerk (= Studien und Texte zur Kirchengeschichte und Geschichte, Fünfte Reihe, 1), Wien

Seitz 1998: $\quad$ Elisabeth Seitz, Primus Truber - Schöpfer der slovenischen Schriftsprache? (= Slavistische Beiträge 363), München

Stabéj 1971: Jože Stabéj, Slowenisch-lateinisches-deutsches (zum Teil) Wörterbuch der Grammatik von Adam Bohorič, in: Adam Bohorič, Arcticae horulae. Die erste Grammatik der slowenischen Sprache, Wittenberg 1584. II. Teil: Untersuchungen, München, 144-206

Toporišič 1966: Jože Toporišič, Slovenski knjižni jezik 2, Maribor

Toporišič 1983/84: Jože Toporišič, O Trubarjevi pisavi, pravorečju in pravopisu, Jezik in slovstvo 29, 226-232

Toporišič 1986: Jože Toporišič, Bohoričica 16. stoletja, in: 16. stoletje v slovenskem jeziku, književnosti in kulturi, uredila B. Pogorelec s sodelovanjem J. Koruze (= Obdobja 6), Ljubljana, 271-305

Toporišič Jože 1987: Jože Toporišič, Adam Bohorizh, Arcticae horulae succisivae - Zimske urice proste. Prevedel in spremno študijo napisal Jože Toporišič, Maribor

Toporišič 1992: $\quad$ Jože Toporišič, Kritične pripombe k Trubarjevemu delu s strani slovenskih besedoljubov in kritikov, Slavistična revija 40, 241-254

Gerhard Neweklowsky

Emil-Mende-Gasse 15

A-9073 Klagenfurt

gerhard.neweklowsky@univie.ac.at 
